https://idp.uoc.edu

ARTÍCULO

\title{
Una aproximación a las limitaciones participativas del civic crowdfunding
}

\author{
Bruno González-Cacheda \\ Universidad de Vigo
}

Fecha de presentación: diciembre de 2020

Fecha de aceptación: abril de 2021

Fecha de publicación: octubre de 2021

\section{Resumen}

En la presente investigación analizamos la dimensión participativa del civic crowdfunding. El objetivo principal es determinar las características y el perfil participativo de la ciudadanía que, a través de aportaciones, contribuye a la financiación de iniciativas de motivación social y cívica. Para ello realizamos un análisis descriptivo a partir de ocho variables que recogen información sobre las particularidades sociodemográficas, las fuentes de información clave para la participación, la experiencia participativa y el grado de conocimiento previo de la entidad promotora. Las variables de estudio se aplican en una muestra de 46 individuos que participaron en alguno de los 27 proyectos de civic crowdfunding y matchfunding de colaboración público-privada alojados en la plataforma Goteo, e incluidos en los programas Crowdfunding ZGZ 2017 y Conjuntament. Desarrollo Económico de Proximidad 2018, promovidos por los Ayuntamientos de Zaragoza y Barcelona. Los resultados muestran un participante tipo conformado de forma hegemónica por personas de género femenino, altamente formadas, con un nivel de renta superior a la media, próximas al colectivo promotor de la causa financiable y con experiencia participativa previa a nivel social, cívico y político. Como conclusión debemos señalar las limitaciones del civic crowdfunding como instrumento participativo para la integración de individuos y grupos sociales ajenos a los procesos sociales y cívicos.

\section{Palabras clave}

participación, integración, civic crowdfunding, matchfunding, público-privado 


\title{
An approach to the participative limitations of civic crowdfunding
}

\begin{abstract}
In this research we analyse the commitment dimension of civic crowdfunding. The main objective is to determine the characteristics and the citizen involvement profile which, through donations, contribute to the financing of social and civic motivation initiatives. To do this, we carry out a descriptive analysis based on eight variables that collect information on sociodemographic particularities, key information sources for participation, involvement experience and the degree of prior knowledge of the promoting entity. The study variables are based on a sample of 46 individuals who participated in any of the 27 civic crowdfunding and matchfunding projects of public-private collaboration hosted on the Goteo platform, and included in the Crowdfunding ZGZ 2017 and Conjuntament Desarrollo Económico de Proximidad 2018 programs that were promoted by the municipalities of Zaragoza and Barcelona. The results show a type of participant formed hegemonically by adult individuals, highly educated, with an income level above the average, close to the group that promotes the fundable cause and with previous involvement experience at the social, civic and political level. In conclusion, we must point out the limitations of civic crowdfunding as a participatory instrument for the integration of individuals and social groups outside of social and civic processes.
\end{abstract}

\section{Keywords}

participation, integration, civic crowdfunding, matchfunding, public-private 


\section{Introducción}

El auge y expansión del crowdfunding como mecanismo para la financiación de proyectos e iniciativas de naturaleza empresarial y mercantil ha atraído en los últimos años una importante y creciente atención en el ámbito académico a través de diversas publicaciones e investigaciones. Sin embargo, el uso de dicha herramienta para la financiación de causas cívicas, sociales y políticas ha generado un interés sensiblemente menor. Así, y tras una búsqueda a través de las palabras clave civic crowdfunding y matchfunding, fueron seleccionados diez artículos académicos relacionados con nuestro objeto de estudio. La revisión de la literatura hallada revela una importante insuficiencia en el análisis de las campañas de financiación desde el punto de vista de sus propiedades intrínsecamente participativas. Con la finalidad de mejorar e incrementar el conocimiento acerca de las características y el perfil de las personas que participan en proyectos de civic crowdfunding, hemos seleccionado diversos proyectos promovidos dentro de las convocatorias de matchfunding impulsadas bajo el paraguas de la plataforma Goteo.

El matchfunding, una fórmula implementada por la plataforma Goteo desde el año 2016 e incluida dentro de la categoría civic crowdfunding, implica la colaboración públicoprivada de diversas instituciones públicas con entidades de corte cívico que promueven proyectos relacionados con distintos ámbitos, pero unidos por el interés social de la finalidad de la causa financiable. Para el análisis de los participantes en los proyectos de matchfunding, hemos seleccionado los programas liderados por los Ayuntamientos de Zaragoza y Barcelona, desarrollados durante los años 2017 y 2018, denominados Crowdfunding ZGZ 2017 y Conjuntament. Desarrollo Económico de Proximidad 2018. En total, bajo la cobertura de dichos programas, han sido lanzadas un total de 27 campañas de financiación. A partir de la información contenida en la plataforma Goteo, relativa a cada uno de los 27 proyectos, hemos obtenido una muestra derivada de la respuesta a un cuestionario por parte de 46 individuos que, a través de donaciones económicas, participaron en la financiación de dichas iniciativas. Del reducido tamaño de la muestra, derivado de la dificultad para acceder a los datos personales y de contacto de las personas participantes, se deriva la principal limitación de nuestra investigación. Este aspecto debe ser solventado en futuros estudios que mejoren y amplíen la muestra obtenida.
Antes de presentar los resultados, la discusión y las conclusiones relacionadas con el perfil participativo de las personas encuestadas, en los siguientes epígrafes nos acercaremos a las premisas teóricas y a los hallazgos realizados hasta el momento relacionados con el civic crowdfunding y la participación digital. En función de la revisión teórica y científica, estableceremos la pregunta de investigación y las variables de estudio, y detallaremos los materiales y métodos utilizados.

\section{Civic crowdfunding y matchfunding}

Más allá de la diversidad asociada al concepto de crowdfunding, en las distintas definiciones podemos observar una serie de elementos comunes (Jovanovic, 2019; Sedlitzky y Franz, 2019). En primer lugar, el rol central atribuido a internet como elemento que determina la configuración flexible y descentralizada de los proyectos de crowdfunding. En segundo lugar, la naturaleza de las aportaciones financieras en función del retorno derivado de las mismas, orientado a la obtención de beneficios o motivados por otro tipo de causas. Sobre la base de este criterio podemos dividir los proyectos en dos grandes grupos: el crowdfunding de inversiones y el de donaciones. En tercer y último lugar, es subrayada la composición sobre la que se articulan y financian los proyectos de crowdfunding, tal y como sostiene Jovanovic (2019, pág. 2): «we argue that crowdfunding is made up of three factors: the people, the project, and the platform: People, fundraisers and funders, come together to propose and support a project on an online platform».

En la presente investigación nos centraremos en el denominado civic crowdfunding. De esta forma, según señala Hamman (2015), los proyectos de civic crowdfunding serían aquellos orientados a crear bienes y servicios en favor de la comunidad, independientemente de la participación o no de los miembros de la comunidad en la financiación de los proyectos (Davies, 2015). Como ya hemos mencionado, otra de las características definitorias de este tipo de proyectos tendría que ver con el papel protagonista de asociaciones, cooperativas y entidades del tercer sector en la promoción e impulso de dichas iniciativas (González-Cacheda, 2018). Para completar la definición de civic crowdfunding parece pertinente tener en cuenta 
las observaciones realizadas por Stiver et al. (2014), en las que ponen de manifiesto que, más allá de la creación de bienes de carácter público, los ingredientes distintivos de este tipo de proyectos tendrían que ver con su articulación a través de redes de cooperación entre ciudadanos, entidades sociales e instituciones y gobiernos locales. A este respecto, una de las experiencias que mayor nivel de éxito y atención académica (Cruz-Maceín y GonzálezAzcárate, 2020; Senabre y Fuster, 2019) ha alcanzado es el denominado matchfunding promovido por la plataforma de financiación en línea Goteo.

El funcionamiento de esta tipología de civic crowdfunding es sencillo. Se inicia con la disposición de un fondo concreto y la fijación de una serie de objetivos asociados a determinados ámbitos específicos de actuación por parte de una institución pública o social. A continuación, y a través de la plataforma Goteo, se realiza una llamada para la presentación de proyectos susceptibles de financiación a todas aquellas entidades y ciudadanos relacionados con los sectores específicos asociados a los ámbitos financiables determinados en las bases de la convocatoria. Una vez recogidas todas las propuestas se procede a la selección de aquellas que, bajo criterios de pertinencia y calidad, formarán parte de la ronda final de financiación. A la fase de selección le sucede un período de capacitación y formación para la mejora de los elementos comunicativos de los proyectos financiables con el fin de mejorar los índices de recaudación. Finalmente, dentro de la última fase del matchfunding y a través de la plataforma Goteo, se activa la campaña de recaudación propiamente dicha. La particularidad de este modelo de civic crowdfunding estriba en la cofinanciación de las propuestas, euro a euro, entre las aportaciones de la ciudadanía y de las instituciones promotoras de los distintos programas de matchfunding, de forma que cada euro aportado en el ámbito de la sociedad civil es complementado por una cantidad idéntica -pero limitada a través de un tope máximo- desde el ámbito institucional (Cruz-Maceín y González-Azcárate, 2020; Senabre y Fuster, 2018; Goteo, 2016).

Esta fórmula presenta unos mejores resultados respecto a la tasa de éxito y a la cifra media de recaudación por proyecto. En concreto, Senabre y Fuster (2018), a partir de la comparación de los datos financieros obtenidos por proyectos de matchfunding y proyectos de crowdfunding general financiados en Goteo, registran un diferencial positivo del $18 \%$ en la tasa de éxito y una suma superior a los dos mil euros de media en favor de las iniciativas financiadas a través de la primera de las modalidades. Por el contrario, cabe subrayar que los proyectos de matchfunding obtienen un menor respaldo (56\%) en la media de apoyos individuales, hecho que se ve compensado por las aportaciones efectuadas desde las instituciones públicas y sociales a través del mecanismo de cofinanciación. En la misma línea, cabe subrayar el estudio realizado por Hong y Ryu (2019), en el que confirman el mayor rendimiento financiero de los proyectos financiados mediante la colaboración público-privada como consecuencia de la participación de instituciones públicas. Estas jugarían un papel clave para el aumento de los niveles de información y confianza entre los potenciales financiadores, y, por consiguiente, en los resultados financieros de los proyectos.

Respecto a las propiedades participativas del civic crowdfunding, en el siguiente epígrafe nos centraremos en la controversia académica relativa al alcance real y el impacto informativo, por un lado, y a las ventajas y desventajas que, desde un punto de vista participativo, aparecen asociadas a internet en general y al civic crowdfunding en particular.

\section{La dimensión informativa y participativa del civic crowdfunding}

Como hemos señalado en el anterior apartado, la investigación académica relacionada con las potencialidades de internet y las herramientas digitales para la información y la participación ha dado lugar a resultados discordantes y a un debate polarizado entre autores que defienden sus virtudes y otros que enumeran sus defectos. Según Haro y Sampedro (2011), la red permite a las organizaciones sociales la coordinación de acciones directas, la distribución de información, la movilización de recursos y la articulación de múltiples tipos de campañas a distancia y de manera instantánea. En el mismo sentido, Gil de Zúñiga,

1. Las campañas de matchfunding desarrolladas por la plataforma Goteo siguen el modelo reward. Esta fórmula ofrece una recompensa de carácter material a todas aquellas personas que realicen una aportación financiera. 
Jung y Valenzuela (2012) subrayan la eficacia de las redes sociales en su dimensión informativa.

Desde el punto de vista participativo, y en conexión con la reducción de costes señalada en el anterior parágrafo, internet ejercería un rol incentivador para el crecimiento de determinadas formas de compromiso (Anduiza, Cantijoch y Gallego, 2009). Además, según Borge, Cardenal y Malpica (2012), el hecho de poseer habilidades digitales sería la cuestión clave para el aumento de la participación incluso en ciudadanos con bajos niveles de interés social o político. Por otra parte, la proliferación de dispositivos digitales y la conexión en red estaría generando un incremento de nuevas formas de adscripción y participación personalizada y ad hoc. De esta forma, individuos refractarios a la vinculación colectiva tradicional estarían prestando apoyo a determinadas causas y proyectos de forma puntual y selectiva (Bennett y Segerberg, 2012).

Desde una perspectiva que introduce un mayor nivel de crítica en torno a las características y propiedades de internet para la información y la participación, Blumler (2016) advierte una fragmentación en las esferas públicas emergentes, y una configuración sobre elementos que potencian el carácter homofílico y endogámico como consecuencia de la exposición selectiva que caracteriza a los espacios digitales orientados a la suministración de información. Además, tal y como sostienen Van Aelst et al. (2017), la motivación previa de los usuarios de internet determinaría el impacto comunicativo de las nuevas esferas públicas, dando como resultado un aumento en las desigualdades en la exposición informativa y el conocimiento de la realidad entre grupos sociales en función de dicha variable.

Pero el elemento motivacional no sería, según diversos estudios, el único elemento clave para la información y la participación digital. Según Schlozman, Verba y Brady (2010), los recursos individuales, en forma de tiempo, dinero y habilidades, jugarían un papel fundamental como variable explicativa para el compromiso social y político. Las habilidades relacionadas con la participación online aparecerían asociadas, en primer lugar, a nivel formativo y educacional (Van Deursen et al., 2017). En segundo lugar, tal y como sostienen Hoffmann y Lutz (2019), conviene tener en cuenta la edad como un elemento de exclusión asociado a la denominada «brecha digital». Este fenómeno afectaría fundamentalmente a los ciudadanos de mayor edad, menos familiarizados con el manejo de herramientas digitales.

Asimismo, es necesario prestar atención, como variable explicativa, al nivel de capital social (Putnam, 2000) y a la posición social de los individuos desde un punto de vista relacional. Como sostienen Ferrer, Medina y Torcal (2006) la disposición de conexiones y recursos puede condicionar positivamente la participación y el compromiso. Así, la pertenencia, el contacto o la proximidad a las redes conformadas por organizaciones, colectivos o entidades sociales proporciona información y conocimiento, al tiempo que fomenta e incentiva determinadas actitudes y comportamientos cívicos a través del incremento de los niveles de confianza social. En relación con esta idea, Hoffmann y Lutz (2019) subrayan el posible aumento de las asimetrías participativas generadas por internet y los canales digitales, como consecuencia de la mayor actividad de aquellos grupos sociales tradicionalmente participativos, afectando de forma negativa a colectivos que, como las mujeres, gozan de una menor inserción en el ámbito y en las redes públicas y sociales.

Sobre la base de los determinantes señalados en las anteriores líneas, relacionados con la motivación, las habilidades, los recursos, la experiencia previa y los niveles de capital social y relacional, autores como Norris (2001) o Van Laer (2010) ponen de manifiesto la posibilidad del aumento de las desigualdades participativas a partir de un crecimiento del repertorio participativo de los perfiles activistas. A este respecto Van Laer (2010) señala, sobre la base de la denominada «hipótesis de refuerzo», que la participación digital estaría protagonizada fundamentalmente por ciudadanos activos, procedentes del entorno de colectivos o grupos organizados, con experiencia adquirida a través de la participación en múltiples causas a lo largo del tiempo (Jäske y Ertiö, 2019).

En relación con el civic crowdfunding, diversas investigaciones (Sedlitzky y Franz, 2019; Davies, 2015; Stiver et al., 2014) han puesto de manifiesto sus características inherentemente participativas. De tal forma, que el éxito de los proyectos financiables depende del apoyo de la ciudadanía, que a través de aportaciones monetarias ejerce su capacidad electiva dando viabilidad en su caso a los proyectos propuestos. Según Davies (2015), la participación en proyectos de civic crowdfunding estaría determinada por tres factores fundamentales. En primer lugar, por el acce- 
so efectivo a la plataforma o aplicación tecnológica. En segundo lugar, y asociado a la brecha digital, la disposición de un nivel suficiente de habilidades para el desarrollo de actividades regulares a través de internet y de dispositivos electrónicos para la conexión a la red (Jäske y Ertiö, 2019). En este sentido ha sido comentado como problemático el desempeño a través de canales digitales de los grupos de personas de mayor edad (Hoffmann y Lutz, 2019). Entre las variables explicativas de carácter sociodemográfico, y para un correcto análisis de la participación digital, diversos autores subrayan la importancia del nivel educativo de los individuos (Sedlitzky y Franz, 2019; Van Deursen et al., 2017). En tercer y último lugar, Davies (2015) señala la importancia del nivel de recursos financieros en la medida en que la participación por medio de civic crowdfunding se produce a través de una aportación monetaria. La naturaleza socioeconómica de este tipo de participación puede dar lugar a una relación directa entre el grado de influencia y el nivel de renta.

Para Sedlitzky y Franz (2019), aunque la brecha digital es una realidad a tener en cuenta, el civic crowdfunding facilitaría la participación de aquellas personas con dificultades para participar de forma presencial o de aquellos grupos de ciudadanía tradicionalmente menos activos en la toma de decisiones, como los adolescentes o los adultos jóvenes. Dentro de las potencialidades participativas del matchfunding, Cruz-Maceín y González-Azcárate (2020) destacan la capacidad de dicha herramienta para la conexión entre la ciudadanía y las instituciones públicas a través de la canalización de las demandas ciudadanas expresadas mediante el apoyo financiero a determinadas causas y proyectos. Por su parte, Stiver et al. (2014) inciden en la capacidad de comunicación y conexión de las redes sociales utilizadas en campaña para la creación de relaciones colaborativas y participativas alrededor de los proyectos de civic crowdfunding.

Una vez apuntadas las características, así como los posibles inconvenientes y posibilidades del civic crowdfunding como mecanismo participativo, y con la finalidad de aumentar el conocimiento relativo a la potencialidad participativa e inclusiva del civic crowdfunding, en los siguientes epígrafes trataremos de dar respuesta a la siguiente pregunta: ¿Cuál es el perfil sociodemográfico, la experiencia participativa y la relación con las entidades promotoras de los individuos que realizan aportaciones a proyectos de civic crowdfunding?

\section{Metodología}

Para responder a la pregunta planteada en el anterior apartado, realizamos un análisis descriptivo a través de la implementación de un breve cuestionario online realizado a través de la plataforma Eval\&Go, articulado a través de diez preguntas cerradas, y orientado a obtener información procedente de aquellos individuos que mediante aportaciones monetarias participaron en proyectos de civic crowdfunding en algún momento de su vida. Para la obtención de la muestra de estudio, y ante la imposibilidad de conocer el universo de estudio, fueron seleccionados un total de 27 proyectos de matchfunding alojados en la plataforma Goteo, única en España en la promoción específica de proyectos público-privados. Estas iniciativas aparecen integradas dentro de los dos programas de ámbito municipal finalizados cuando se realizó el estudio: Crowdfunding ZGZ 2016 y 2017 y Conjuntament. Desarrollo Económico de Proximidad 2018, coparticipados por los Ayuntamientos de Zaragoza y Barcelona respectivamente. Sobre la base de la información ofrecida por Goteo en su portal web, relativa a cada uno de los proyectos, pudieron ser identificados con nombre y apellidos un total de 453 personas participantes. Posteriormente, y con la finalidad de hacer llegar el cuestionario a los participantes registrados, se estableció contacto a través de diferentes redes sociales. En total, fue posible hacer llegar el cuestionario a 162 personas, de las cuales 46 lo respondieron de forma completa $(28,3 \%)$. Por lo tanto, el muestreo realizado fue de carácter no probabilístico.

\begin{tabular}{|l|l|}
\hline ACRÓNIMO & \multicolumn{1}{c|}{ VARIABLE } \\
& \\
\hline EDAD & Rango de edad de los participantes \\
SEXO & Sexo de las personas participantes \\
ESTUDIOS & Nivel de estudios reglados \\
RENTA & Nivel de renta neto anual \\
INFO & Fuente de información para la participación \\
COPR & Conocimiento previo de la entidad promotora \\
COLPRO & Colaboración previa con la entidad promotora \\
EXPAR & Experiencia en actividades participativas \\
\hline Nota. Elaboración propia \\
\hline
\end{tabular}

Tabla 1. Variables de estudio 
Las variables de estudio que aparecen recogidas en la tabla 1 resumen la información recogida en el cuestionario. Para el perfilado y la caracterización de las personas participantes en los proyectos de matchfunding analizamos la EDAD, el SEXO, el nivel de ESTUDIOS y la RENTA. Además, y con el objetivo de conocer las fuentes de información de los individuos que realizan aportaciones a los 27 proyectos seleccionados, sintetizamos a través de la variable INFO los distintos emisores relevantes que desarrollaron actividades comunicativas durante la campaña de recaudación. Para el análisis de la relación de las personas participantes con la entidad promotora, agrupamos los datos acerca del conocimiento previo y el nivel de colaboración en las actividades desarrolladas a través de las variables COPR y COLPRO. Finalmente, en la tabla 1 presentamos la variable EXPAR, que recoge y sintetiza información referente a la experiencia participativa en el plano social y político de los individuos consultados.

Antes de presentar los gráficos de los resultados obtenidos, realizadas con la herramienta Excel, debemos señalar las limitaciones de la presente investigación, derivadas del reducido tamaño de la muestra obtenida, consecuencia de la dificultad para acceder y contactar con las personas participantes en los proyectos de civic crowdfunding y matchfunding.

\section{Resultados}

En este apartado presentaremos los resultados derivados de la investigación llevada a cabo a través del cuestionario realizado a las personas participantes en proyectos de civic crowdfunding y matchfunding. Para ello, en primer lugar, mostraremos diversos gráficos que recogen las características sociodemográficas de las personas que respondieron al cuestionario. A continuación, ofreceremos datos relativos a los canales de comunicación clave para la participación. Y, finalmente, recogeremos los gráficos que muestran la relación y el nivel de proximidad con las entidades promotoras de los proyectos y la experiencia participativa de las personas encuestadas.

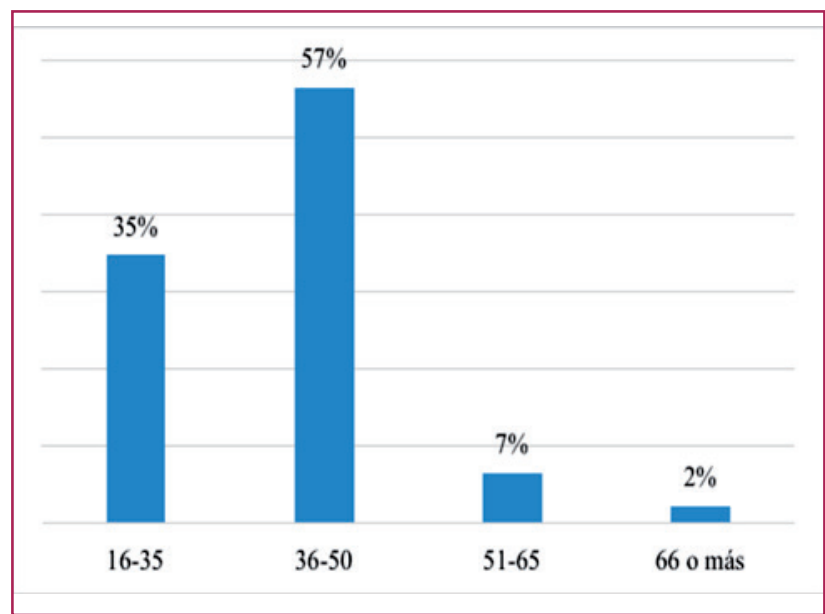

Gráfico 1. Rango de edad de las personas que respondieron al cuestionario. Elaboración propia para la variable EDAD

Tal y como se puede observar en el gráfico 1, el grueso de la participación en proyectos civic crowdfunding, a partir de las respuestas al cuestionario, se concentra en personas de edad adulta, de forma que el $57 \%$ se encuentra en el rango de edad que va desde los 36 a los 50 años. A continuación, encontramos al grupo de participantes de menor edad, situados en el rango que abarca a las personas de 16 a 35 años (35\%). Por último, cabe señalar la escasa respuesta de personas de más de 50 años, con un $9 \%$ en total (ver gráfico 1).

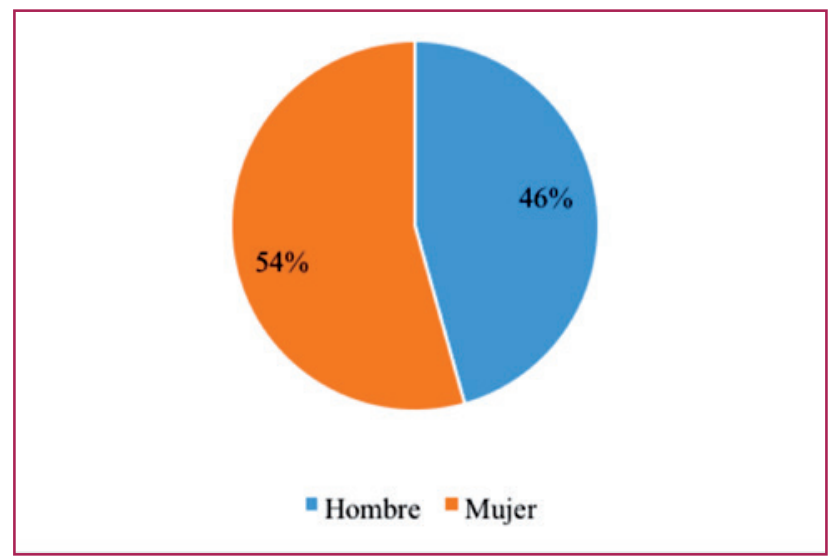

Gráfico 2. Sexo de las personas que respondieron al cuestionario (SEXO). Elaboración propia para la variable SEXO 
En lo relativo al sexo de las personas participantes, y aunque podemos hablar de cierto equilibrio, en este caso el número de mujeres que respondieron al cuestionario y que afirmaron participar en proyectos de civic crowdfunding fue superior al número de hombres, con un 54\% frente a un $46 \%$ del total (ver gráfico 2).

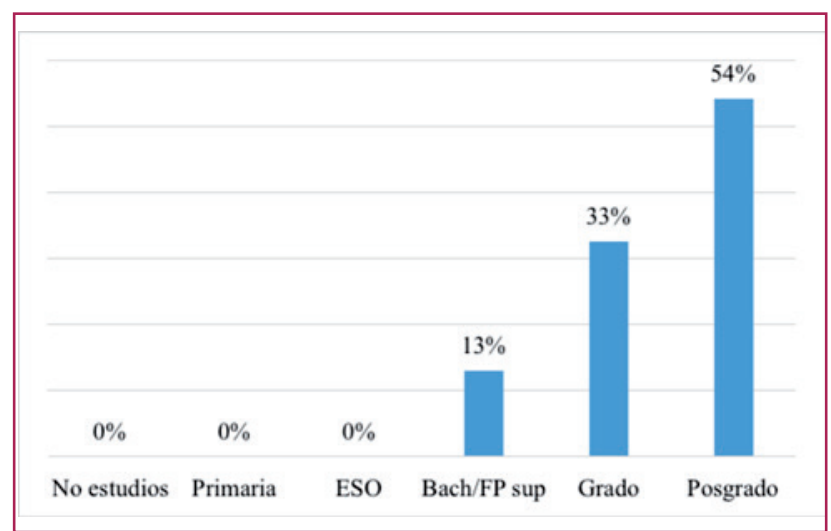

Gráfico 3. Nivel de estudios reglados de las personas que respondieron al cuestionario. Elaboración propia para la variable ESTUDIOS

Respecto al nivel de formación reglada alcanzada por las personas participantes, podemos observar una alta concentración en las categorías universitarias de grado y posgrado, representando entre ambas un $87 \%$. A continuación, y con un $13 \%$ del total, aparecen aquellos individuos que completaron estudios de formación profesional superior y bachillerato. Tal y como se puede ver en el gráfico 3, destaca la ausencia de personas con niveles de educación primaria, secundaria obligatoria e individuos sin formación reglada.

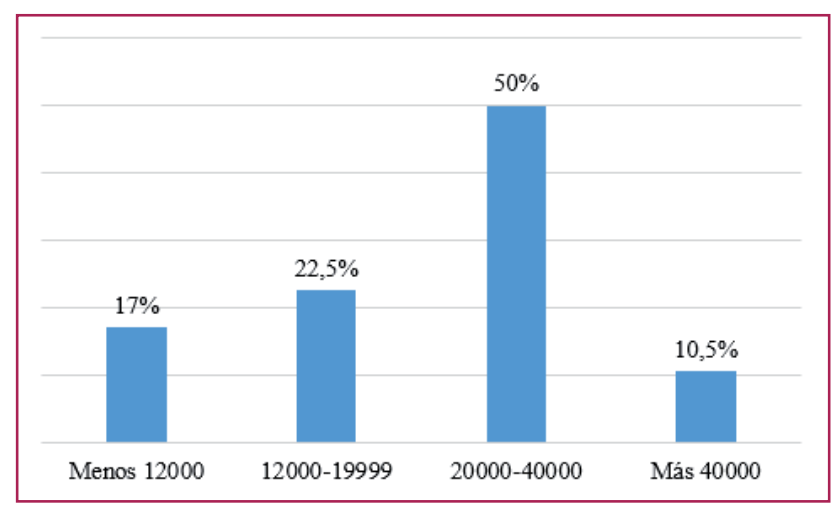

Gráfico 4. Nivel de renta neta anual de las personas que respondieron al cuestionario. Elaboración propia para la variable RENTA
Si observamos el nivel de renta neta personal percibida a lo largo de una anualidad, encontramos que un 50\% de las personas participantes obtienen un nivel de ingresos que se sitúa en el rango que va desde los 20.000 a los 40.000 euros. A continuación, podemos ver un $22,5 \%$ de participantes que afirma haber ingresado en la última anualidad una cantidad que oscila entre los 12.000 y los 19.999 euros. Dentro del rango inferior, encontramos a un $17 \%$ que responde haber obtenido unos ingresos inferiores a los 12.000 euros. Mientras que, finalmente, y en el extremo opuesto, un $10,5 \%$ de los participantes afirma haber percibido unos ingresos netos superiores a 40.000 euros (ver gráfico 4).

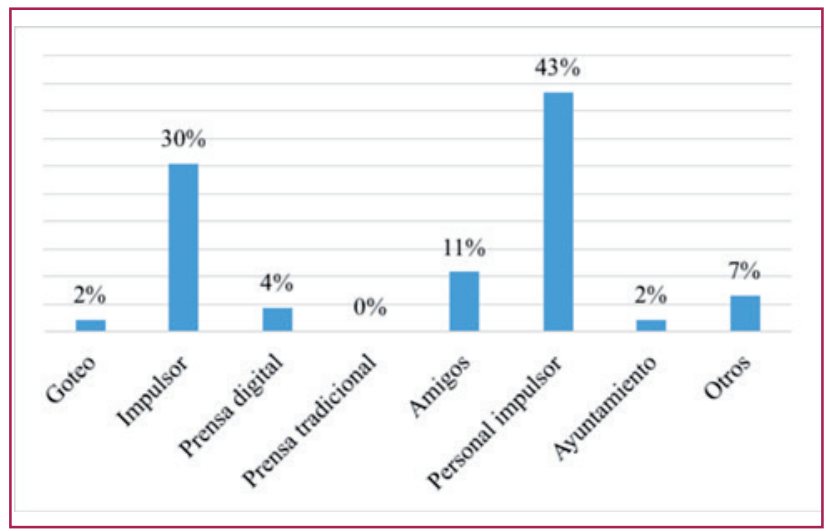

Gráfico 5. Fuente o canal emisor de la información clave para la participación. Elaboración propia para la variable INFO

Como vía principal para el acceso a la información necesaria para la realización de aportaciones financieras, encontramos el contacto y la comunicación del personal vinculado a la entidad impulsora de los proyectos, de forma que el $43 \%$ de las personas participantes conocieron la iniciativa financiable a través de este canal. El segundo canal más relevante, aparece vinculado a la actividad comunicativa realizada de forma directa por la entidad promotora (30\%). Más allá de la entidad promotora y su entorno, encontramos un $11 \%$ de participantes que afirman haberse enterado de la campaña financiable a través de la información difundida por amigos y conocidos en redes de comunicación social. Finalmente, y a una importante distancia, aparecen como fuentes de información para la participación los canales de la plataforma Goteo (2\%), las noticias difundidas a través de prensa digital (4\%) y los 
canales de información institucional de los Ayuntamientos de Zaragoza y Barcelona (2\%) (ver gráfico 5).

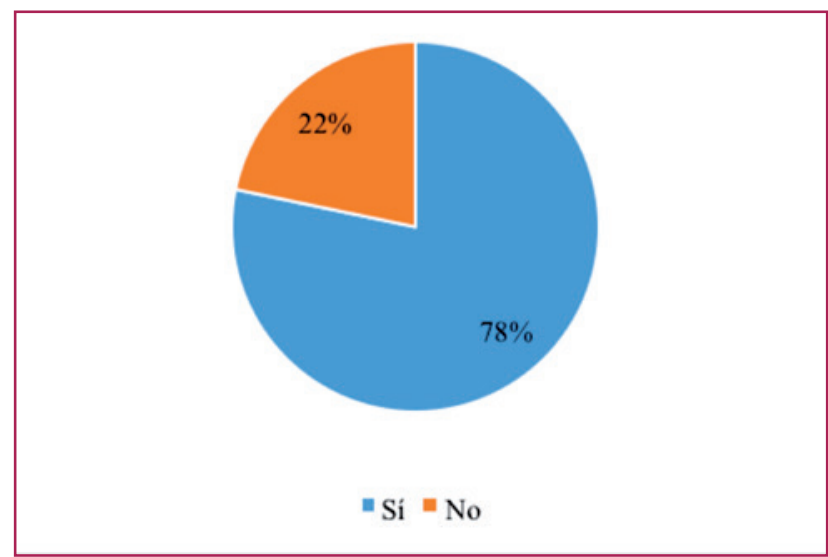

Gráfico 6. Nivel de conocimiento previo de la entidad promotora. Elaboración propia para la variable COPR

Respecto al grado de conocimiento previo de la entidad o colectivo que busca financiación, por parte de las personas participantes, los datos parecen concordar con los presentados en el gráfico 5, en el que una gran parte de los financiadores afirmaban haberse informado a través de la propia entidad promotora o de sus integrantes. Así, un $78 \%$ de los financiadores señala tener conocimiento previo a la donación de la existencia y actividades de la entidad impulsora de la campaña (ver gráfico 6).

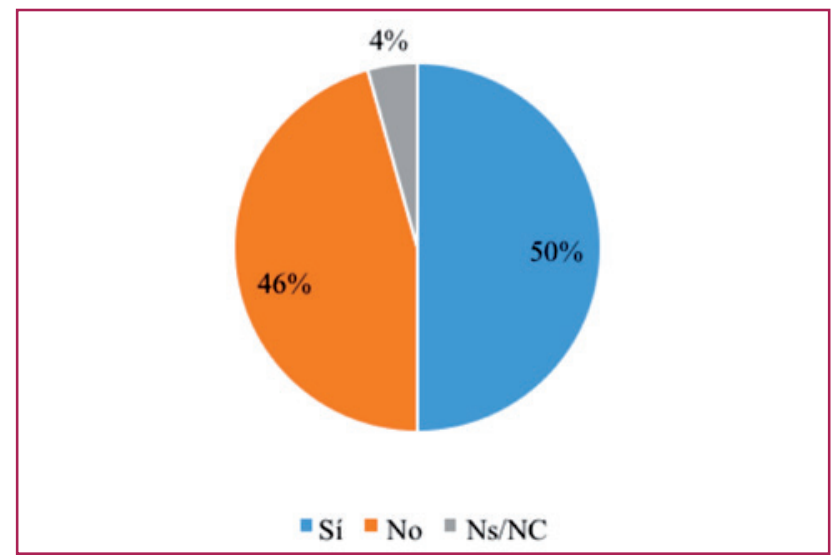

Gráfico 7. Participación y colaboración con la entidad promotora. Elaboración propia para la variable COLPRO
Además del alto índice de conocimiento previo señalado en el gráfico 6, la información detallada en el gráfico 7 comporta un importante porcentaje (50\%) de participantes y donantes que colaboran regular o puntualmente con la entidad que lidera la campaña de financiación de civic crowdfunding. En el polo opuesto, aparece un 46\% de personas que afirman no participar en dichas actividades (ver gráfico 7).

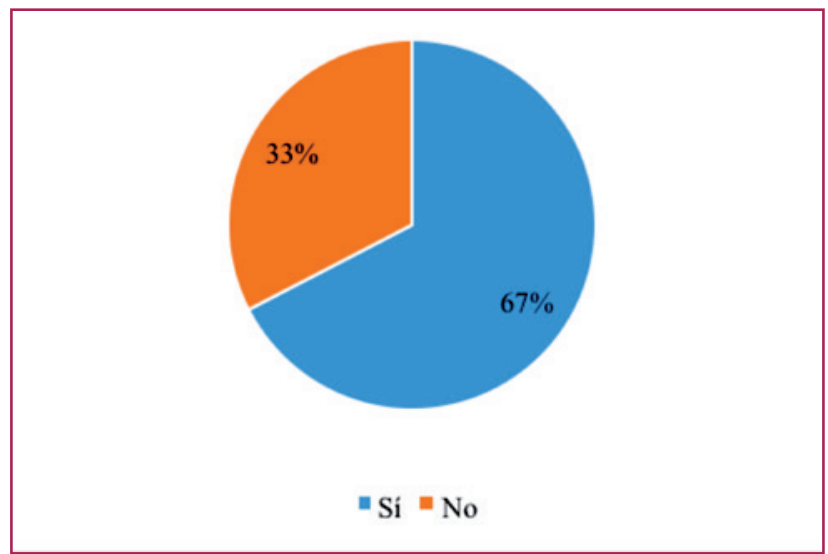

Gráfico 8. Participación en actividades sociales, cívicas y políticas. Elaboración propia para la variable EXPAR

Respecto al compromiso y la experiencia participativa en actividades de carácter sociopolítico, promovidas por asociaciones, ONG, movimientos sociales, sindicatos, cooperativas o partidos políticos, tal y como se recoge en el gráfico 8 , se puede observar un importante volumen (67\%) de donantes en los proyectos de civic crowfunding analizados que participa o participó activamente de la forma señalada.

En definitiva, podemos describir la participación tipo en los proyectos de civic crowdfunding como mayoritariamente femenina, conformada por personas de mediana edad, alto nivel formativo y experiencia participativa previa. En el siguiente epígrafe, sobre la base de la información detallada en los gráficos comentados y la evidencia científica publicada hasta el momento, analizaremos y discutiremos las implicaciones de dichos resultados sobre la naturaleza participativa del civic crowdfunding. 


\section{Discusión}

La aparición de internet y la expansión de las aplicaciones digitales y de los dispositivos electrónicos móviles ha traído consigo un intenso debate acerca de la potencialidad de dichos instrumentos para la expansión de las actividades participativas en distintos ámbitos de la sociedad y de la Administración pública. Más allá de la evidente introducción de un mayor grado de eficiencia comunicativa, diversas investigaciones (Jäske y Ertiö, 2019; Van Laer, 2010) han subrayado las limitaciones como instrumento para la integración participativa de determinados grupos socioeconómicos ajenos a los procesos colectivos y sociales.

En la presente investigación hemos tratado de aproximarnos a las características participativas y a la composición social y económica de las personas que a través de pequeñas aportaciones económicas colaboran y participan en proyectos de civic crowdfunding y matchfunding de carácter público-privado. Respecto a los recursos disponibles, y a partir de los datos extraídos de los estadísticos descriptivos manejados en el anterior apartado, podemos señalar que el nivel de renta (RENTA) parece situarse como un elemento previo y necesario para la realización de donaciones a través de las iniciativas analizadas (Schlozman, Verba y Brady, 2010). De esta forma, un 67\% de las personas encuestadas afirman percibir un salario neto anual superior a la media estatal, que se sitúa cerca de Ios 12.000 euros (INE, 2020). Este hecho parece confirmar la asociación entre nivel de renta y nivel de influencia en este tipo de mecanismos participativos (Davies, 2015). Más allá de la relación entre renta y participación, debemos contemplar los posibles beneficios de las acciones financiadas sobre colectivos vulnerables o en una posición de desventaja social. En todo caso, los efectos redistributivos e inclusivos de los proyectos financiados a través de civic crowdfunding deben ser examinados exhaustivamente en futuras investigaciones.

Otro elemento determinante y multidimensional para la participación tiene que ver con las habilidades técnicas y los procesos cognitivos relacionados con la disposición para tomar parte en cuestiones de carácter colectivo. Los datos relativos al nivel educativo de las personas participantes en los proyectos estudiados presentan un $87 \%$ de casos con estudios universitarios de grado o posgrado. Las cifras recogidas a través de la variable ESTUDIOS coinciden, así pues, con investigaciones previas que vinculan la participación digital con el nivel formativo reglado (Sedlitzky y Franz, 2019; Van Deursen et al., 2017). Otra variable relevante relacionada con las habilidades necesarias para la participación digital es la EDAD. Tal y como sostienen Hoffmann y Lutz (2019) uno de los mayores desafíos asociados a los instrumentos para la participación digital tiene que ver con la brecha digital y la exclusión de las personas de mayor edad (Jäske y Ertiö, 2019). En el caso del matchfunding los datos parecen respaldar las tesis de los autores citados anteriormente, de tal forma que únicamente el $9 \%$ de las respuestas al cuestionario proceden de personas de más de cincuenta años.

Por contra, y en sentido opuesto a la evidencia manejada por autores como Hoffmann y Lutz (2019) o Van Deursen et al. (2017), los datos asociados a la variable SEXO muestran una mayor presencia femenina en la participación de los proyectos analizados. El perfil de participación femenino se configura a través de personas de edad joven, alto nivel de estudios e integradas social y laboralmente. En este punto, debemos subrayar la posible conexión entre la participación femenina y las ventajas que ofrece la configuración espacio-temporal de internet para la conciliación de la vida laboral, profesional y sociopolítica de las mujeres.

Tras el análisis de las características sociodemográficas, y en relación con las fuentes de información clave para la participación, los datos revelan el rol clave de la entidad promotora y de su red más directa, conformada por personas relacionadas a través de lazos fuertes. Así, el 73\% de las personas consultadas afirma haber conocido la iniciativa financiada partir del contacto o de las redes sociales de los miembros y de la entidad promotora. En sentido contrario, los canales de comunicación más abiertos muestran una baja eficacia, hecho que denota un importante nivel de endogamia y homofilia de los espacios de comunicación articulados para la difusión de las campañas (Van Aelst et al., 2017; Blumler, 2016). De forma que únicamente el $8 \%$ participó gracias a la difusión realizada desde el ámbito municipal, la plataforma intermediaria Goteo o el ámbito periodístico tradicional o digital.

En sintonía con los resultados mostrados por la variable INFO, las cifras de la variable COPR señalan que un $78 \%$ de las personas que respondieron al cuestionario conocían con anterioridad a la realización de la donación a la entidad promotora de la campaña. Además, un 50\% ma- 
nifestó haber colaborado en las actividades desarrolladas por dicha entidad (COLPRO). El conjunto de la información presentada por las variables INFO, COPR y COLPRO ponen de manifiesto la centralidad de la entidad promotora como fuente del conocimiento, del capital social y de la confianza necesaria para el compromiso y la participación a través de aportaciones monetarias (Ferrer, Medina y Torcal, 2006; Putnam, 2000). Por el contrario, y al hilo de los datos, la plataforma intermediaria o las administraciones públicas parecen jugar un papel secundario respecto a los elementos anteriormente mencionados. El déficit comunicativo de los Ayuntamientos de Barcelona y Zaragoza supone uno de los principales problemas para la participación efectiva de la ciudadanía en los proyectos analizados. En la medida en que la participación aparece condicionada por los niveles de información disponibles, consideramos necesario el desarrollo de un plan de comunicación institucional y una mayor coordinación con el resto de actores que conforman las campañas, a fin de dar a conocer los programas de civic crowdfunding entre el conjunto de la ciudadanía.

Finalmente, los rasgos y las características discutidas hasta aquí, junto a los datos presentados por la variable EXPAR, que recoge que hasta un $67 \%$ de las personas encuestadas participan o han participado en actividades de naturaleza social, cívica o política, dibujan un retrato general de las personas analizadas asociado a las ideas de multiactivismo o pluriparticipación (Hoffmann y Lutz, 2019; Van Laer, 2010). De esta forma, podemos afirmar que la participación en proyectos de civic crowdfunding y matchfunding está protagonizada por ciudadanos con experiencia participativa en múltiples causas y colectivos de distinta naturaleza a lo largo del tiempo (Jäske y Ertiö, 2019). Así, frente a las tesis movilizadoras de los canales y aplicaciones digitales (Borge, Cardenal y Malpica, 2012; Anduiza, Cantijoch y Gallego, 2009), los hallazgos produ- cidos se sitúan cerca de la hipótesis de refuerzo (Norris, 2001).

\section{Conclusión}

En la presente investigación nos hemos centrado en aportar información sobre algunas claves de la dimensión participativa del civic crowdfunding. Con todas las precauciones que se derivan del reducido tamaño de la muestra, las variables de análisis muestran un perfil participativo conformado mayoritariamente por individuos adultos, altamente formados y con un nivel de renta neta anual superior a la media. La procedencia y los canales de información que sirvieron como fuente para la participación indican una elevada proximidad a la entidad promotora y una escasa integración de personas ajenas a la misma y a los procesos de participación social, cívica y política. De este modo, y en sintonía con los teóricos defensores de la hipótesis de refuerzo, consideramos que la participación a través de civic crowdfunding y matchfunding puede estar ensanchando la brecha participativa entre la ciudadanía informada y participativa y los grupos e individuos excluidos y ajenos a los procesos sociales, cívicos y políticos.

En todo caso, el enfoque adoptado y las limitaciones asociadas al tamaño de la muestra dejan abiertas diversas cuestiones de análisis para futuras investigaciones. Entre otras señalaremos las siguientes: el estudio de la motivación de las personas que realizan donaciones a proyectos de civic crowdfunding y una profundización en el análisis participativo en función de la variable género; el examen en profundidad de la estrategia informativa y comunicativa de los distintos actores involucrados en los programas de matchfunding, y el estudio del impacto y de las consecuencias sociales de los proyectos financiados a través de matchfunding. 


\section{Referencias bibliográficas}

ANDUIZA, E.; CANTIJOCH, M.; GALLEGO, A. (2009). «Political participation and the Internet: A field essay». Information, Communication \& Society, núm. 12, págs. 860-878 [en línea] DOI: https://doi. org/10.1080/13691180802282720 [Fecha de consulta: 27 de abril de 2021].

BENNETT, W. L.; SEGERBERG, A. (2012). «The logic of connective action: Digital media and the personalization of contentious politics». Information, communication \& society, núm. 15, págs. 739-768 [en línea] DOI: https://doi.org/10.1080/1369118X.2012.670661 [Fecha de consulta: 27 de abril de 2021].

BORGE, R.; CARDENAL, A. S.; MALPICA, C. (2012). «El impacto de Internet en la participación política: Revisando el papel del interés político». Arbor, núm. 188, págs. 733-750 [en línea] DOI: https://doi. org/10.1080/1369118X.2012.670661 [Fecha de consulta: 27 de abril de 2021].

BLUMLER, J. G. (2016). «The fourth age of political communication». Politiques de communication, núm. 6, págs. 19-30 [en línea] DOI: https://doi.org/10.1002/9781118541555.wbiepc213 [Fecha de consulta: 27 de abril de 2021].

CRUZ-MACEÍN, J. L.; GONZÁLEZ-AZCÁRATE, M. (2020). «Innovación social y políticas públicas locales: matchfunding, ¿una nueva herramienta para el desarrollo local?». Serie Estudios IMIDRA, núm. 5, págs. 1-39 [en línea] https://www.comunidad.madrid/sites/default/files/matchfunding_y_politicas_ publicas_locales_serie_estudios_imidra_5.pdf [Fecha de consulta: 27 de abril de 2021].

DAVIES, R. (2014). Civic crowdfunding: Participatory communities, entrepreneurs and the political economy of place (Masters). Cambridge, MA: MIT. DOI: https://doi.org/10.2139/ssrn.2434615 [Fecha de consulta: 27 de abril de 2021].

DAVIES, R. (2015). «Three provocations for civic crowdfunding». Information, Communication \& Society, núm. 18, págs. 342-355 [en línea] DOI: https://doi.org/10.1080/1369118X.2014.989878 [Fecha de consulta: 27 de abril de 2021].

FERRER, M.; MEDINA, L. E.; TORCAL, M. (2006). «La participación política: factores explicativos». En: MONTERO, J. R.; FONT, J.; TORCAL, M. (eds.). Ciudadanos, asociaciones y participación en España. Madrid: CIS.

GIL DE ZÚÑIGA, H.; JUNG, N.; VALENZUELA, S. (2012). «Social media use for news and individuals' social capital, civic engagement and political participation». Journal of computer-mediated communication, núm. 17, págs. 319-336 [en línea] DOI: https://doi.org/10.1111/j.1083-6101.2012.01574.x [Fecha de consulta: 27 de abril de 2021].

GONZÁLEZ-CACHEDA, B. (2018). «Social innovation and crisis in the third sector in Spain. Results, challenges and limitations of "Civic Crowdfunding"». Journal of Civil Society, núm. 14, págs. 275-291 [en línea] DOI: https://doi.org/10.1080/17448689.2018.1459239 [Fecha de consulta: 27 de abril de 2021].

GOTEO (2016). «Matchfunding: crowdfunding bajo el principio de corresponsabilidad. Fundación Goteo [en línea] http://fundacion.goteo.org/blog/matchfunding-crowdfunding-bajo-el-principio-decorresponsabilidad [Fecha de consulta: 27 de abril de 2021].

HARO, C.; SAMPEDRO, V. (2011). «Activismo político en Red: Del movimiento por la vivienda digna al 15M». Teknokultura, núm. 8, págs. 167-185 [en línea] https://revistas.ucm.es/index.php/TEKN/article/view/48025 [Fecha de consulta: 27 de abril de 2021].

HAMMAN, E. (2015). «Save the reef: Civic crowdfunding and public interest environmental litigation». QUT Law Review, núm. 15, págs. 159-173 [en línea] DOI: https://doi.org/10.5204/qutlr.v15i1.604 [Fecha de consulta: 27 de abril de 2021]. 
HOFFMANN, C. P.; LUTZ, C. (2019). «Digital divides in political participation: The mediating role of social media self efficacy and privacy concerns». Policy \& Internet, págs. 1-24 [en línea] DOI: https://doi.org/10.1002/poi3.225 [Fecha de consulta: 27 de abril de 2021].

HONG, S.; RYU, J. (2019). «Crowdfunding public projects: Collaborative governance for achieving citizen co-funding of public goods». Government Information Quarterly, núm. 36, págs. 145-153 [en línea] DOI: https://doi.org/10.1016/j.giq.2018.11.009 [Fecha de consulta: 27 de abril de 2021].

INSTITUTO NACIONAL DE ESTADÍSTICA (2020). INEbase. Renta anual media [en línea] https://www.ine. es/jaxiT3/Tabla.htm?t=9947 [Fecha de consulta: 27 de abril de 2021].

JÄSKE, M.; ERTIÖ, T. (2019). «The democratic potential of civic applications». Information Polity, núm. 24, págs. 1-19 [en línea] DOI: https://doi.org/10.3233/IP-180105 [Fecha de consulta: 27 de abril de 2021].

JOVANOVIC, T. (2019). «Crowdfunding: What do we know so far?». International Journal of Innovation and Technology Management, núm. 16, págs. 1950009 [en línea] DOI: https://doi.org/10.1142/ S0219877019500093 [Fecha de consulta: 27 de abril de 2021].

NORRIS, P. (2001). Digital divide: civic engagement, information poverty, and the Internet Worldwide. Cambridge: Cambridge University Press. DOI: https://doi.org/10.1017/CB09781139164887 [Fecha de consulta: 27 de abril de 2021].

PUTNAM, R. (2000). Solo en la bolera. Colapso y resurgimiento de la comunidad americana. Barcelona: Galaxia Gutenberg.

SEDLITZKY, R.; FRANZ, Y. (2019). «"What if we all chip in?" Civic crowdfunding as alternative financing for urban development projects». Built Environment, núm. 45, págs. 26-44. [en línea] DOI: https://doi.org/10.2148/benv.45.1.26 [Fecha de consulta: 27 de abril de 2021].

SENABRE, E.; FUSTER, M. (2019). «Match-funding as a formula for crowdfunding». SSRN. [en línea] doi. https://doi.org/10.1145/3233391.3233967 [Fecha de consulta: 27 de abril de 2021].

SCHLOZMAN, K. L.; VERBA, S.; BRADY, H. E. (2010). «Weapon of the strong? Participatory inequality and the Internet». Perspectives on Politics, núm. 8, págs. 487-509 [en línea] DOI: https://doi. org/10.1017/S1537592710001210 [Fecha de consulta: 27 de abril de 2021].

STIVER, A.; BARROCA, L.; MINOCHA, S.; RICHARDS, M.; ROBERTS, D. (2014). «Civic crowdfunding research: Challenges, opportunities, and future agenda». New media \& society, núm. 17, págs. 249-271 [en línea] DOI: https://doi.org/10.1177/1461444814558914 [Fecha de consulta: 27 de abril de 2021].

VAN AELST, P. et al. (2017). «Political communication in a high-choice media environment: a challenge for democracy?». Annals of the International Communication Association, núm. 41, págs. 3-27. [en línea] DOI: https://doi.org/10.1080/23808985.2017.1288551 [Fecha de consulta: 27 de abril de 2021].

VAN DEURSEN, A. J.; HELSPER, E.; EYNON, R.; VAN DIJK, J. A. (2017). «The compoundness and sequentiality of digital inequality». International Journal of Communication, núm. 11, págs. 452-473 [en línea] https://ijoc.org/index.php/ijoc/article/view/5739 [Fecha de consulta: 27 de abril de 2021].

VAN LAER, J. (2010). «Activists online and offline: The internet as an information channel for protest demonstrations». Mobilization: An International Quarterly, núm. 15, págs. 347-366 [en línea] DOI: https://doi.org/10.17813/maiq.15.3.8028585100245801 [Fecha de consulta: 27 de abril de 2021]. 


\title{
Cita recomendada
}

GONZÁLEZ-CACHEDA, Bruno (2021). «Una aproximación a las limitaciones participativas del civic crowdfunding». IDP. Revista de Internet, Derecho y Política, núm. 33 (octubre). UOC [Fecha de consulta: dd/mm/aa] http://dx.doi.org/10.7238/idp.v0i33.377280

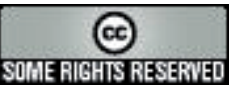

\begin{abstract}
Los textos publicados en esta revista están -si no se indica lo contrario- bajo una licencia Reconocimiento-Sin obras derivadas 3.0 España de Creative Commons. Puede copiarlos, distribuirlos y comunicarlos públicamente siempre que cite su autor y la revista y la institución que los publica (IDP. Revista de Internet, Derecho y Política; UOC); no haga con ellos obras derivadas. La licencia completa se puede consultar en: http://creativecommons.org/ licenses/by-nd/3.0/es/deed.es.
\end{abstract}

\section{Sobre el autor}

Bruno González-Cacheda

bruno.gonzalez.cacheda@uvigo.es

Área de Ciencia Política de la Universidade de Vigo

Profesor interino del Área de Ciencia Política y de la Administración en la Universidade de Vigo. Doctorando del Programa en Creatividade e Innovación Social e Sostible (UVigo). Máster en Políticas Públicas y Sociales (UPF/JHU) y en Políticas Comunitarias y Cooperación Territorial (UVigo/UMinho). Licenciado en Ciencias Políticas y de la Administración (USC) y diplomado en Gestión y Administración Pública (UVigo). Autor de más de una decena de publicaciones en revistas y obras colectivas de ámbito nacional e internacional relacionadas con diversas temáticas, entre las que destacan:

GONZÁLEZ-CACHEDA, B. (2018). «Social innovation and crisis in the third sector in Spain. Results, challenges and limitations of "Civic Crowdfunding"». Journal of Civil Society, núm. 14, págs. 275-291 [en línea] DOI: https://doi.org/10.1080/17448689.2018.1459239 [Fecha de consulta: 27 de abril de 2021].

RAMOS DÍAZ, J.; GONZÁLEZ, B. (2016). «Crowdfunding and Employment: An analysis of the employment effects of crowdfunding in Spain». En: BRÜNTJE, D.; Gajda, O. (eds.). Crowdfunding in Europe: State of the Art in Theory and Practice. Springer. DOI: https://doi.org/10.1007/978-3-319-18017-5_7 [Fecha de consulta: 27 de abril de 2021]. 